\title{
Searching for dark photons in hyperon decays
}

\author{
Jhih-Ying $\mathrm{Su}^{1}$ and Jusak Tandean $\odot^{1,2}$ \\ ${ }^{1}$ Department of Physics, National Taiwan University, Taipei 106, Taiwan \\ ${ }^{2}$ Physics Division, National Center for Theoretical Sciences, Hsinchu 300, Taiwan
}

(Received 9 December 2019; accepted 30 January 2020; published 28 February 2020)

\begin{abstract}
That massless dark photons could exist and have flavor-changing magnetic-dipole interactions with down-type light quarks is an attractive possibility which may be realized in various new-physics scenarios. It is potentially testable not only in kaon processes but also via two-body hyperon decays involving missing energy carried away by the massless dark photon. We explore the latter within a simplified model approach and take into account constraints from the kaon sector. We find that the branching fractions of some of these hyperon modes are allowed to be as high as a few times $10^{-4}$. Such numbers are likely to be within the sensitivity reaches of ongoing experiments like BESIII and future ones at super charm-tau factories.
\end{abstract}

DOI: 10.1103/PhysRevD.101.035044

Models of new physics (NP) beyond the standard model (SM) may have a dark sector containing an extra Abelian gauge group, $\mathrm{U}(1)_{D}$, under which all SM fields are singlets. This symmetry may be broken spontaneously or unbroken, causing the associated gauge boson, the dark photon, to get mass or stay massless. These possibilities are appealing for a number of reasons and have been much studied in the past [1-14]. They have motivated many dedicated hunts for dark photons in recent years [13-20], with negative outcomes so far. The majority of these efforts have been based on the assumption that $\mathrm{U}(1)_{D}$ is broken and the accompanying massive dark photon, $A^{\prime}$, can interact directly with SM fermions through a renormalizable operator $\epsilon e A_{\mu}^{\prime} J_{\mathrm{EM}}^{\mu}$, with $e J_{\mathrm{EM}}$ being the electromagnetic current and the small parameter $\epsilon$ originating from kinetic mixing between the dark and SM Abelian gauge fields [12-14]. This entails that $A^{\prime}$ could be produced in the scattering or decays of SM particles, including the decays of unflavored mesons, and it could decay into electrically charged fermions or mesons and perhaps also invisibly into other dark particles, allowing experiments to set limits on $\epsilon$ over various ranges of the $A^{\prime}$ mass [13-20].

If instead $\mathrm{U}(1)_{D}$ remains unbroken, its associated gauge boson will not acquire any mass. In this case, there is always a linear combination of the dark and SM Abelian gauge fields which does not have renormalizable couplings to SM members and which can be identified with the

Published by the American Physical Society under the terms of the Creative Commons Attribution 4.0 International license. Further distribution of this work must maintain attribution to the author(s) and the published article's title, journal citation, and DOI. Funded by SCOAP ${ }^{3}$. massless dark photon [1,2]. Thus, the latter, which we denote by $\bar{\gamma}$, has no direct interactions with SM fermions, unlike the massive one. As a consequence, $\bar{\gamma}$ can escape the restrictions implied by the aforementioned quests for $A^{\prime}$. Nevertheless, since $\bar{\gamma}$ can still exert influence on the SM via higher-dimensional operators generated by loop diagrams involving particles which are charged under $\mathrm{U}(1)_{D}$ and also coupled to SM fields [2-4], more efficient ways to look for it may be available and hence should be pursued. Here we propose some of them, which apply to the fairly general situation described in the following.

We concentrate on flavor-changing neutral current (FCNC) effects arising from the massless dark photon having nonrenormalizable interactions with the $d$ and $s$ quarks. This might bring to mind kaon processes which for the massive dark photon could serve as good probes, especially the decays $K \rightarrow \pi A^{\prime}$ and $K^{+} \rightarrow \mu^{+} \nu A^{\prime}$ [9-12]. However, with $\bar{\gamma}$ being massless and not interacting directly with SM fermions, $K \rightarrow \pi \bar{\gamma}$ is forbidden by angularmomentum conservation and gauge invariance and $\mathrm{K}^{+} \rightarrow$ $\mu^{+} \nu \bar{\gamma}$ would have a very suppressed rate. Rather, in this massless case, observing $K^{+} \rightarrow \pi^{+} \pi^{0} \bar{\gamma}$ has been suggested as an avenue to study the $d s \bar{\gamma}$ coupling [4].

In this paper, we would like to show that the hyperon sector potentially offers a competitive window to access $s \rightarrow d \bar{\gamma}$. Generally the quark transition $s \rightarrow d \not{E}$ gives rise to the FCNC decays of hyperons into a lighter baryon plus missing energy $(\mathscr{E})$ which is carried away by one or more invisible particles. If these are a pair of spin- $1 / 2$ fermions or spinless bosons, it is presently possible for NP to enhance the branching fractions of the hyperon modes considerably above the SM expectations to levels discoverable by ongoing or future searches [21,22]. Particularly, new results on these decays may be forthcoming from the 
BESIII experiment [23,24], supplying information on potential NP in $s \rightarrow d \boldsymbol{E}$ complementary to that gained from quests for their kaon counterparts. If the missing energy is carried away by a massless dark photon instead, the final state has only two particles, and consequently the prospects for BESIII pursuing these two-body modes are expected to be comparatively better as long as their branching fractions are not too small. As we will see shortly, they can indeed be sizable in some scenarios recently proposed in the literature, implying that the two-body decays may be testable with the existing hyperon data and upcoming measurements.

In the main processes under consideration a massless dark photon, $\bar{\gamma}$, is emitted on-shell due to its dipole-type flavor-changing couplings to the $d$ and $s$ quarks brought about by loop diagrams involving heavy nonstandard particles. This is described by the dimension-five operators in the effective Lagrangian

$$
\mathcal{L}_{d s \bar{\gamma}}=-\bar{d} \sigma^{\mu \nu}\left(\mathbb{C}+\gamma_{5} \mathbb{C}_{5}\right) s \bar{F}_{\mu \nu}+\text { H.c. }
$$

where $\mathbb{C}$ and $\mathbb{C}_{5}$ are constants having the dimension of inverse mass and dependent on the details of the underlying NP model, $\sigma^{\mu \nu}=(i / 2)\left[\gamma^{\mu}, \gamma^{\nu}\right]$, and $\bar{F}_{\mu \nu}=\partial_{\mu} \bar{A}_{\nu}-$ $\partial_{\nu} \bar{A}_{\mu}$ denotes the field strength tensor of $\bar{\gamma}$.
Our hyperon decays of interest are $\mathfrak{B} \rightarrow \mathfrak{B}^{\prime} \bar{\gamma}$ with $\mathfrak{B} \mathfrak{B}^{\prime}=\Lambda n, \Sigma^{+} p, \Xi^{0} \Lambda, \Xi^{0} \Sigma^{0}, \Xi^{-} \Sigma^{-}$, all of the baryons having spin $1 / 2$, and $\Omega^{-} \rightarrow \Xi^{-} \bar{\gamma}$, where $\Omega^{-}$has spin $3 / 2$. For these modes, to calculate the baryonic parts of the amplitudes induced by $\mathcal{L}_{d s \bar{\gamma}}$, we need to know the matrix elements $\left\langle\mathfrak{B}^{\prime}\left|\bar{d} \sigma^{\mu \nu}\left(1, \gamma_{5}\right) s\right| \mathfrak{B}\right\rangle$ and $\left\langle\Xi^{-}\left|\bar{d} \sigma^{\mu \nu}\left(1, \gamma_{5}\right) s\right| \Omega^{-}\right\rangle$ contracted with the dark photon's momentum $\bar{q}$ and polarization $\bar{\varepsilon}$, which satisfy the gauge condition $\bar{\varepsilon} \cdot \bar{q}=0$. We additionally have $\bar{q}^{2}=0$ due to $\bar{\gamma}$ being on-shell and $\bar{q}=p_{\mathfrak{B}}-p_{\mathfrak{B}^{\prime}}=p_{\Omega}-p_{\Xi}$, the momentum difference between the initial and final baryons. It follows that we can express

$$
\begin{gathered}
\left\langle\mathfrak{B}^{\prime}\left|\bar{d} i \sigma^{\mu \nu}\left(\mathbb{C}+\gamma_{5} \mathbb{C}_{5}\right) s\right| \mathfrak{B}\right\rangle \bar{\varepsilon}_{\mu}^{*} \bar{q}_{\nu} \\
=\frac{f_{\mathfrak{B}^{\prime} \mathfrak{B}}}{2} \bar{u}_{\mathfrak{B}^{\prime}}\left(\bar{q}^{*} \not{q} \mid-\not \bar{q} \bar{q}^{*}\right)\left(\mathbb{C}+\gamma_{5} \mathbb{C}_{5}\right) u_{\mathfrak{B}}, \\
\left\langle\Xi^{-}\left|\bar{d} i \sigma^{\mu \nu}\left(\mathbb{C}+\gamma_{5} \mathbb{C}_{5}\right) s\right| \Omega^{-}\right\rangle \bar{\varepsilon}_{\mu}^{*} \bar{q}_{\nu} \\
=\bar{u}_{\Xi}\left(\frac{f_{\Xi \Omega}}{2}+\frac{\tilde{f}_{\Xi \Omega \Omega} \not \not_{\Omega}}{2 m_{\Omega^{-}}}\right)\left(\bar{q}^{*} \bar{q}_{\tau}-\not \bar{q} \bar{\varepsilon}_{\tau}^{*}\right)\left(\gamma_{5} \mathbb{C}+\mathbb{C}_{5}\right) u_{\Omega}^{\tau},
\end{gathered}
$$

where $f_{\mathfrak{B}^{\prime} \mathfrak{B}}, f_{\Xi \Omega}$, and $\tilde{f}_{\Xi \Omega}$ represent form factors evaluated at $\bar{q}^{2}=0$ and $u_{X}\left(u_{\Omega}^{\mu}\right)$ stands for the Dirac (RaritaSchwinger) spinor of $X\left(\Omega^{-}\right)$. With these formulas, we derive the decay rates, arriving at

$$
\begin{aligned}
\Gamma_{\mathfrak{B} \rightarrow \mathfrak{B}^{\prime} \bar{\gamma}} & =\frac{\Delta_{\mathfrak{B} \mathfrak{B}^{\prime}}^{6} f_{\mathfrak{B}^{\prime} \mathfrak{B}}^{2}}{2 \pi m_{\mathfrak{B}}^{3}}\left(|\mathbb{C}|^{2}+\left|\mathbb{C}_{5}\right|^{2}\right), \\
\Gamma_{\Omega^{-} \rightarrow \Xi^{-} \bar{\gamma}} & =\frac{4 \Delta_{\Omega^{-} \Xi^{-}}^{6}\left(f_{\Xi \Omega^{2}} m_{\Omega^{-}}+\tilde{f}_{\Xi \Omega \Omega} m_{\Xi^{-}}\right)^{2}+\Delta_{\Omega^{-} \Xi^{-}}^{8}\left(\tilde{f}_{\Xi \Omega}^{2}-f_{\Xi \Omega}^{2}\right)}{96 \pi m_{\Omega^{-}}^{5}}\left(|\mathbb{C}|^{2}+\left|\mathbb{C}_{5}\right|^{2}\right),
\end{aligned}
$$

where $\Delta_{X Y}=\sqrt{m_{X}^{2}-m_{Y}^{2}}$. As regards $f_{\mathfrak{B}^{\prime} \mathfrak{B},} f_{\Xi \Omega}$ and $\tilde{f}_{\Xi \Omega}$, it turns out that matrix elements analogous to those in Eqs. (2) and (3) were estimated a while ago in an investigation [25] on the contributions of the transition $s \rightarrow d \gamma$ to hyperon radiative weak decays, $\mathfrak{B} \rightarrow \mathfrak{B}^{\prime} \gamma$ and $\Omega^{-} \rightarrow \Xi^{-} \gamma$, involving the ordinary photon. ${ }^{1}$ With appropriate changes, we can apply the results found therein [25] to the corresponding processes with a massless dark photon, thus obtaining

$f_{\mathfrak{B}^{\prime} \mathfrak{B}}=\mathcal{C}_{\mathfrak{B}^{\prime} \mathfrak{B}} \sqrt{\frac{m_{\mathfrak{B}^{\prime}}}{m_{\mathfrak{B}}}}, \quad f_{\Xi \Omega}=\tilde{f}_{\Xi \Omega}=\frac{4 \sqrt{m_{\Omega} m_{\Xi}}}{m_{\Omega}+m_{\Xi}}$.

For all of these modes, $\mathfrak{B}_{\mathrm{i}} \rightarrow \mathfrak{B}_{\mathrm{f}} \bar{\gamma}$, combining Eq. (5) with Eq. (4) we can write the rates as

\footnotetext{
${ }^{1}$ It is worth noting that (2) and (3) can be shown to be consistent in form with the general gauge-invariant amplitudes known in the literature for these hyperon radiative weak decays [26-29].
}

$$
\Gamma_{\mathfrak{B}_{\mathrm{i}} \rightarrow \mathfrak{B}_{\mathrm{f}} \bar{\gamma}}=\frac{\Delta_{\mathfrak{B}_{\mathrm{i}} \mathfrak{B}_{\mathrm{f}}}^{6} \mathcal{C}_{\mathfrak{B}_{\mathrm{f}} \mathfrak{B}_{\mathrm{i}}}^{2} m_{\mathfrak{B}_{\mathrm{f}}}}{2 \pi m_{\mathfrak{B}_{\mathrm{i}}}^{4}}\left(|\mathbb{C}|^{2}+\left|\mathbb{C}_{5}\right|^{2}\right),
$$

and in Table I we have listed the values of $\mathcal{C}_{\mathfrak{B}_{\mathrm{f}} \mathfrak{B}_{\mathrm{i}}}^{2}$, which were determined in Ref. [25] with quark-model SU(6) wave functions and for which we have assumed overlap factors of 1 .

Since $\mathbb{C}$ and $\mathbb{C}_{5}$ are generally independent of each other, for simplicity hereafter we assume that $\mathbb{C}_{5}$ is absent, which is also the case in the particular examples we look at below. For each of the hyperon modes, we can then

TABLE I. Values of $\mathcal{C}_{\mathfrak{B}_{\mathrm{f}} \mathfrak{B}_{\mathrm{i}}}^{2}$ in Eq. (6) for $\mathfrak{B}_{\mathrm{f}} \mathfrak{B}_{\mathrm{i}}=n \Lambda, p \Sigma^{+}$, $\Lambda \Xi^{0}, \Sigma^{0} \Xi^{0}, \Sigma^{-} \Xi^{-}, \Xi^{-} \Omega^{-}$from Ref. [25].

\begin{tabular}{lcccccc}
\hline \hline $\mathfrak{B}_{\mathrm{f}} \mathfrak{B}_{\mathrm{i}}$ & $n \Lambda$ & $p \Sigma^{+}$ & $\Lambda \Xi^{0}$ & $\Sigma^{0} \Xi^{0}$ & $\Sigma^{-} \Xi^{-}$ & $\Xi^{-} \Omega^{-}$ \\
\hline $\mathcal{C}_{\mathfrak{B}_{\mathrm{f}} \mathfrak{B}_{\mathrm{i}}}^{2}$ & $\frac{3}{2}$ & $\frac{1}{9}$ & $\frac{1}{6}$ & $\frac{25}{18}$ & $\frac{25}{9}$ & $\frac{4}{3}$ \\
\hline \hline
\end{tabular}


TABLE II. The branching fractions $\mathcal{B}$ of $\mathfrak{B}_{\mathrm{i}} \rightarrow \mathfrak{B}_{\mathrm{f}} \bar{\gamma}$ divided by $|\mathbb{C}|^{2}$ in the $\mathbb{C}_{5}=0$ case.

\begin{tabular}{ccccccc}
\hline \hline Decay mode & $\Lambda \rightarrow n \bar{\gamma}$ & $\Sigma^{+} \rightarrow p \bar{\gamma}$ & $\Xi^{0} \rightarrow \Lambda \bar{\gamma}$ & $\Xi^{0} \rightarrow \Sigma^{0} \bar{\gamma}$ & $\Xi^{-} \rightarrow \Sigma^{-} \bar{\gamma}$ & $\Omega^{-} \rightarrow \Xi^{-} \bar{\gamma}$ \\
\hline $\mathcal{B}$ & $2.75 \times 10^{12}$ & $1.54 \times 10^{11}$ & $4.95 \times 10^{11}$ & $1.12 \times 10^{12}$ & $1.32 \times 10^{12}$ & $5.18 \times 10^{12}$ \\
\hline \hline$|\mathbb{C}|^{2}$ & & & & & \\
\hline \hline
\end{tabular}

evaluate the corresponding branching fraction $\mathcal{B}\left(\mathfrak{B}_{\mathrm{i}} \rightarrow\right.$ $\left.\mathfrak{B}_{\mathrm{f}} \bar{\gamma}\right)$ once $\mathbb{C}$ is numerically given. For later convenient use, we have collected in Table II the numbers for $\mathcal{B}\left(\mathfrak{B}_{\mathrm{i}} \rightarrow\right.$ $\left.\mathfrak{B}_{\mathrm{f}} \bar{\gamma}\right)$ divided by $|\mathbb{C}|^{2}$, after employing the measured masses from Ref. [30]. In light of the successful quarkmodel predictions of other baryonic quantities which rely on the same assumption of a single-quark operator transforming like a component of the quark spin [25], we expect that the estimates displayed in this table are good to within factors of 2 .

To see how large $\mathcal{B}\left(\mathfrak{B}_{\mathrm{i}} \rightarrow \mathfrak{B}_{\mathrm{f}} \bar{\gamma}\right)$ may be at present without restrictions from hyperon data, we now consider, as benchmarks, different maximal values of $|\mathbb{C}|$ in two scenarios recently discussed in the literature. Although they are variations of the same simplified model of NP, the two different sets of predictions which they make will serve to illustrate how hyperon and kaon measurements together could help distinguish various NP possibilities. Therefore, similar analyses could be performed for other models. In both of these scenarios, proposed in Refs. [4,5], the $d s \bar{\gamma}$ interactions originate from loop diagrams involving new particles comprising massive fermions which are SM singlets as well as heavy scalar bosons which are triplets under color SU(3) and some of which are doublets under the $\mathrm{SM} \mathrm{SU}(2)_{L}$. The new fermions and bosons are all charged under $\mathrm{U}(1)_{D}$ and have Yukawa-like interactions with the $d$ and $s$ quarks, which allows the dimension-five operator for $s \rightarrow d \bar{\gamma}$ to be constructed. Since our aim here is to examine the implications of this coupling for the hyperon modes, once it has been subject to constraints from other sectors, we will not dwell further on the details of the underlying NP model. Rather, we will simply take most of the relevant results provided in Refs. [4,5] at face value and employ them to compute the hyperon decay rates.

In the first scenario the $d s \bar{\gamma}$ coupling constant is given by [4]

$$
\mathbb{C}=\frac{e_{D} \xi}{64 \pi^{2} \tilde{\Lambda}}
$$

where $e_{D}$ parametrizes the dark photon's interaction strength, $\xi$ is a product of two common Yukawa couplings between the new particles and SM quarks, and $\tilde{\Lambda}$ is the effective heavy mass scale of the dark sector. The Yukawa interactions also bring about box-diagram contributions to the kaon-mixing quantity $\Delta m_{K}$. Their impact can be numerically expressed in terms of the ratio $\xi / \tilde{\Lambda}$ as [4]

$$
\Delta m_{K}^{\mathrm{NP}}=8.47 \times 10^{-13} \mathrm{TeV}^{3} \frac{\xi^{2}}{\tilde{\Lambda}^{2}},
$$

which is dominated by the effects of four-quark operators that have chirally enhanced matrix elements between the $K^{0}$ and $\bar{K}^{0}$ states. To constrain $\xi / \tilde{\Lambda}$, the new contribution in Eq. (8) is required [4] to be less than $30 \%$ of its experimental counterpart, namely $\Delta m_{K}^{\mathrm{NP}}<0.3 \Delta m_{K}^{\exp }$, where $\Delta m_{K}^{\text {exp }}=3.484 \times 10^{-15} \mathrm{GeV}$ [30]. This constitutes the main restraint on $\xi / \tilde{\Lambda}$. With the size of $e_{D}$ following from the choice $\alpha_{D}=e_{D}^{2} /(4 \pi)=0.1$ [4], putting things together we then obtain from Eq. (7)

$$
|\mathbb{C}|<2.0 \times 10^{-9} \mathrm{GeV}^{-1}
$$

Combining this with the entries in Table II yields the maximal branching fractions $\mathcal{B}_{\max }$ of the hyperon modes shown in the second row of Table III and labeled [I].

In the second scenario the $d s \bar{\gamma}$ coupling constant has the form [5]

$$
\mathbb{C}=\frac{e_{D} \mathcal{D}_{M}}{2 \tilde{\Lambda}},
$$

where $\mathcal{D}_{M}$ contains a product of a couple of Yukawa couplings between the new particles and the $d$ and $s$ quarks and is related to the kaon-mixing parameter by $\Delta m_{K}^{\mathrm{NP}}=32 \pi^{2} \mathcal{D}_{M}^{2} f_{K}^{2} m_{K^{0}} /\left(3 \tilde{\Lambda}^{2}\right)$, with $f_{K}=159.8 \mathrm{MeV}$ being the kaon decay constant and $m_{K^{0}}=497.6 \mathrm{MeV}$ [30]. In this instance, unlike the preceding one, the $K^{0}-\bar{K}^{0}$

TABLE III. The maximal branching fractions of $\mathfrak{B}_{\mathrm{i}} \rightarrow \mathfrak{B}_{\mathrm{f}} \bar{\gamma}$ with the $|\mathbb{C}|$ values from [I] Eq. (9) and [II] Eq. (12).

\begin{tabular}{lcccccc}
\hline \hline Decay mode & $\Lambda \rightarrow n \bar{\gamma}$ & $\Sigma^{+} \rightarrow p \bar{\gamma}$ & $\Xi^{0} \rightarrow \Lambda \bar{\gamma}$ & $\Xi^{0} \rightarrow \Sigma^{0} \bar{\gamma}$ & $\Xi^{-} \rightarrow \Sigma^{-} \bar{\gamma}$ & $\Omega^{-} \rightarrow \Xi^{-} \bar{\gamma}$ \\
\hline $\mathcal{B}_{\max }[\mathrm{I}]$ & $1.1 \times 10^{-5}$ & $6.0 \times 10^{-7}$ & $1.9 \times 10^{-6}$ & $4.3 \times 10^{-6}$ & $5.1 \times 10^{-6}$ & $2.0 \times 10^{-5}$ \\
$\mathcal{B}_{\max }[\mathrm{II}]$ & $6.7 \times 10^{-4}$ & $3.8 \times 10^{-5}$ & $1.2 \times 10^{-4}$ & $2.7 \times 10^{-4}$ & $3.3 \times 10^{-4}$ & $1.3 \times 10^{-3}$ \\
\hline \hline
\end{tabular}


matrix elements of the contributing four-quark operators are not chirally enhanced. To be consistent with the previous example, we demand $\Delta m_{K}^{\mathrm{NP}}<0.3 \Delta m_{K}^{\exp }$, leading to

$$
\frac{\mathcal{D}_{M}^{2}}{\tilde{\Lambda}^{2}}<7.8 \times 10^{-16} \mathrm{GeV}^{-2},
$$

which is a more stringent limit than that found in Ref. [5] from the condition $\Delta m_{K}^{\mathrm{NP}}<\Delta m_{K}^{\mathrm{exp}}$. Applying this to Eq. (10), with $\alpha_{D}=0.1$ as before, we then get

$$
|\mathbb{C}|<1.6 \times 10^{-8} \mathrm{GeV}^{-1}
$$

This translates into the maximal branching fractions $\mathcal{B}_{\max }$ of the hyperon modes listed in the last row of Table III and labeled [II].

As indicated above, the differences between the two sets of predictions in Table III reflect the fact that the details of the underlying NP in the two cases affect kaon mixing differently. It follows that quests for the hyperon modes could come up with information pertinent for testing NP models. The future measurement on $K^{-} \rightarrow \pi^{-} \pi^{0} \bar{\gamma}$, which arises from the same $s \rightarrow d \bar{\gamma}$ transition, would likely be important as well according to Ref. [4].

As pointed out earlier, the hyperon decays may be searched for in the BESIII experiment [23], which has produced copious $\Lambda, \Sigma, \Xi$, and $\Omega$ hyperons [24]. For their FCNC decays with missing energy carried away by a pair of invisible particles, the proposed BESIII sensitivity levels for the branching fractions of $\Lambda \rightarrow n \mathscr{E}, \Sigma^{+} \rightarrow p \mathscr{E}$, $\Xi^{0} \rightarrow \Lambda \mathscr{E}, \Xi^{0} \rightarrow \Sigma^{0} \mathbb{E}$, and $\Omega^{-} \rightarrow \Xi^{-} \mathscr{E}$ are $3 \times 10^{-7}, 4 \times 10^{-7}$, $8 \times 10^{-7}, 9 \times 10^{-7}$, and $2.6 \times 10^{-5}$, respectively [23]. Since our decays of concern, $\mathfrak{B}_{\mathrm{i}} \rightarrow \mathfrak{B}_{\mathrm{f}} \bar{\gamma}$, are two-body ones, BESIII would have greater sensitivity to them, being able to detect them more efficiently than the three-body ones. This is especially consequential for NP models which generate predictions comparable to those displayed in Table III.

As we await possibly upcoming outcomes of the direct searches for $\mathfrak{B}_{\mathrm{i}} \rightarrow \mathfrak{B}_{\mathrm{f}} \bar{\gamma}$ from BESIII and other (future) experiments, it turns out that we can already extract approximate bounds on their branching fractions indirectly from the data on the observed channels quoted by the Particle Data Group [30]. To do so, for each of these decaying hyperons, we subtract from unity the sum of the PDG branching-fraction numbers with their errors (at 2 sigmas) combined in quadrature. Thus, for the yet-unobserved decay modes of $\Lambda, \Sigma^{+}, \Xi^{0}, \Xi^{-}$, and $\Omega^{-}$, we arrive at the upper limits of $1.4 \%, 8.0 \times 10^{-3}$, $3.4 \times 10^{-4}, 8.3 \times 10^{-4}$, and $1.6 \%$, respectively. Evidently, they accommodate most of the corresponding predictions in Table III, the exceptions being the two $\Xi^{0}$ results together in the bottom row. This suggests that the second scenario considered above may already be in tension with the present hyperon data if its $d s \bar{\gamma}$ coupling constant is not significantly lower than the bound in Eq. (12). Had we imposed the weaker condition $\Delta m_{K}^{\mathrm{NP}}<\Delta m_{K}^{\exp }$, the resulting upper values of $|\mathbb{C}|$ in this scenario would be disfavored more strongly.

In conclusion, we have entertained the possibility that massless dark photons exist and have non-negligible flavorchanging dipole-type couplings to down-type light quarks. These kind of interactions induce FCNC hyperon decays into a lighter baryon plus missing energy carried away by the massless dark photon. We demonstrate that these hyperon modes can be a powerful tool to probe the underlying $s \rightarrow d \bar{\gamma}$ transition, perhaps more so than what kaon decays could offer. We illustrate this with a couple of examples from recent studies in the literature which take into account the current restrictions from the kaon sector. Our findings reveal that one of these two scenarios might already be in tension with the indirect bounds on hyperon decays with missing energy inferred from the available hyperon data. Further tests on the hyperon modes will expectedly come from ongoing experiments such as BESIII and future facilities such as super charm-tau factories. In the near future, BESIII will likely be in a position to discover one or more of these hyperon modes or, if not, set stringent limitations on the $d s \bar{\gamma}$ interactions.

\section{ACKNOWLEDGMENTS}

We would like to thank Hai-Bo Li for information on experimental matters. This research was supported in part by the MOST (Grant No. MOST 106-2112-M-002-003-MY3).
[1] B. Holdom, Two U(1)'s and $\epsilon$ charge shifts, Phys. Lett. 166B, 196 (1986).

[2] B. A. Dobrescu, Massless Gauge Bosons Other Than the Photon, Phys. Rev. Lett. 94, 151802 (2005).

[3] E. Gabrielli, B. Mele, M. Raidal, and E. Venturini, FCNC decays of standard model fermions into a dark photon, Phys. Rev. D 94, 115013 (2016).
[4] M. Fabbrichesi, E. Gabrielli, and B. Mele, Hunting Down Massless Dark Photons in Kaon Physics, Phys. Rev. Lett. 119, 031801 (2017).

[5] M. Fabbrichesi and E. Gabrielli, Dark-sector physics in the search for the rare decays $K^{+} \rightarrow \pi^{+} \bar{\nu} \nu$ and $K_{L} \rightarrow \pi^{0} \bar{\nu} \nu$, arXiv:1911.03755. 
[6] L. Ackerman, M. R. Buckley, S. M. Carroll, and M. Kamionkowski, Dark matter and dark radiation, Phys. Rev. D 79, 023519 (2009).

[7] J. Y. Cen, M. He, X. G. He, and G. Li, Scrutinizing a massless dark photon: Basis independence and new observables, arXiv:1807.11363.

[8] M. He, X. G. He, C. K. Huang, and G. Li, Search for a heavy dark photon at future $e^{+} e^{-}$colliders, J. High Energy Phys. 03 (2018) 139.

[9] V. Barger, C. W. Chiang, W. Y. Keung, and D. Marfatia, Constraint on Parity-Violating Muonic Forces, Phys. Rev. Lett. 108, 081802 (2012).

[10] C. W. Chiang and P. Y. Tseng, Probing a dark photon using rare leptonic kaon and pion decays, Phys. Lett. B 767, 289 (2017).

[11] J. R. Batley et al. (NA48/2 Collaboration), Search for the dark photon in $\pi^{0}$ decays, Phys. Lett. B 746, 178 (2015).

[12] M. Pospelov, Secluded U(1) below the weak scale, Phys. Rev. D 80, 095002 (2009).

[13] R. Essig et al., Working group report: New light weakly coupled particles, arXiv:1311.0029.

[14] J. Alexander et al., Dark sectors 2016 workshop: Community report, arXiv:1608.08632.

[15] M. Ablikim et al. (BESIII Collaboration), Dark photon search in the mass range between 1.5 and $3.4 \mathrm{GeV} / c^{2}$, Phys. Lett. B 774, 252 (2017).

[16] R. Aaij et al. (LHCb Collaboration), Search for Dark Photons Produced in $13 \mathrm{TeV} p p$ Collisions, Phys. Rev. Lett. 120, 061801 (2018).

[17] A. Anastasi et al. (KLOE-2 Collaboration), Combined limit on the production of a light gauge boson decaying into $\mu^{+} \mu^{-}$ and $\pi^{+} \pi^{-}$, Phys. Lett. B 784, 336 (2018).
[18] M. Ablikim et al. (BESIII Collaboration), Measurement of $\mathcal{B}\left(J / \psi \rightarrow \eta^{\prime} e^{+} e^{-}\right)$and search for a dark photon, Phys. Rev. D 99, 012013 (2019).

[19] E. C. Gil et al. (NA62 Collaboration), Search for production of an invisible dark photon in $\pi^{0}$ decays, J. High Energy Phys. 05 (2019) 182.

[20] D. Banerjee et al., Dark Matter Search in Missing Energy Events with NA64, Phys. Rev. Lett. 123, 121801 (2019).

[21] J. Tandean, Rare hyperon decays with missing energy, J. High Energy Phys. 04 (2019) 104.

[22] G. Li, J. Y. Su, and J. Tandean, Flavor-changing hyperon decays with light invisible bosons, Phys. Rev. D 100, 075003 (2019).

[23] H. B. Li, Prospects for rare and forbidden hyperon decays at BESIII, Front. Phys. 12, 121301 (2017); Erratum, Front. Phys. 14, 64001 (2019).

[24] M. Ablikim et al. (BESIII Collaboration), White paper on the future physics programme of BESIII, arXiv:1912.05983.

[25] F. J. Gilman and M. B. Wise, Radiative weak decays of baryons as single quark transitions, Phys. Rev. D 19, 976 (1979).

[26] R. E. Behrends, Photon decay of hyperons, Phys. Rev. 111, 1691 (1958).

[27] J. O. Eeg, The process $\Omega \rightarrow \gamma \Xi$ as a possible test of the Penguin diagram, Z. Phys. C 21, 253 (1984).

[28] R. Safadi and P. Singer, $\Omega^{-} \rightarrow \Xi^{-} \gamma$ and $\Omega^{-} \rightarrow \Xi^{-} \ell^{+} \ell^{-}$ decays and the single quark transition model, Phys. Rev. D 37, 697 (1988); Erratum, Phys. Rev. D 42, 1856 (1990).

[29] G. Eilam, A. Ioannisian, and P. Singer, Asymmetry in the decay $\Omega^{-} \rightarrow \Xi^{-} \gamma$, Mod. Phys. Lett. A 11, 2091 (1996).

[30] M. Tanabashi et al. (Particle Data Group), Review of particle physics, Phys. Rev. D 98, 030001 (2018). 